\title{
The efficacy of mycophenolate mofetil in Japanese patients with systemic lupus erythematosus
}

\author{
Mai Kawazoe ${ }^{1}$, Kaichi Kaneko ${ }^{1}$, Shinichi Kawai ${ }^{2}$, Toshihiro Nanki ${ }^{1}$ \\ ${ }^{I}$ Division of Rheumatology, Department of Internal Medicine, School of Medicine, Faculty of Medicine, Toho \\ University, Japan, ${ }^{2}$ Department of Inflammation and Pain Control Research, School of Medicine, Toho University, \\ Japan
}

[Background] Induction therapy for proliferative lupus nephritis (LN) recommended by American college of rheumatology is glucocorticoids with either intravenous cyclophosphamide (IVCY) or mycophenolate mofetil (MMF). Despite the worldwide use of MMF, the use of MMF for LN in Japan was approved in 2015. Therefore there are few reports which evaluating the renal response of MMF in Japanese patients. Moreover, the effect of MMF on nonrenal disease activity has been poorly studied in Japanese population. Here, we conducted a retrospective clinical study to assess the renal and nonrenal efficacy of MMF in Japanese patients with systemic lupus erythematosus (SLE).

[Methods] Adult Japanese SLE patients who visited our hospital from May 2010 to August 2017 were surveyed. Fourteen patients with LN were given MMF and twelve patients received monthly IVCY as an induction therapy. Moreover, we collected twenty patients without LN who took MMF and nineteen patients who took tacrolimus (TAC) for reduction in glucocorticoid dosage. We assessed the therapeutic effects of MMF, IVCY and TAC on renal and nonrenal disease manifestation at 1, 3, and 6 months after starting the treatment, and compare the responses to each therapy.

[Results] Both treatment with MMF and IVCY reduced proteinuria, serum level of anti-dsDNA Ab, SLE Disease Activity Index (SLEDAI) and daily prednisolone dosage, and at 6 months there was no significant differences in MMF group (mean $0.7 \pm 2.2 \mathrm{~g} / \mathrm{gCr}, 10.0 \pm 18.5 \mathrm{IU} / \mathrm{mL}, 4.0 \pm 3.8,10.0 \pm 4.2 \mathrm{mg}$ ) compared to IVCY group (mean $0.5 \pm 0.4$ $\mathrm{g} / \mathrm{gCr}, 10.0 \pm 20.5 \mathrm{IU} / \mathrm{mL}, 2.0 \pm 2.6,12.0 \pm 4.3 \mathrm{mg}$ ), respectively. Similarly, MMF and TAC were both effective to nonrenal disease activity. At 6 months there was no significant difference in serum level of anti-dsDNA Ab, SLEDAI and daily prednisolone dosage in MMF group (mean $10.0 \pm 62.4 \mathrm{IU} / \mathrm{mL}, 2.0 \pm 1.7,9.5 \pm 5.3 \mathrm{mg}$ ) compared to TAC group (mean $13.0 \pm 53.1 \mathrm{IU} / \mathrm{mL}, 2.5 \pm 1.6,10.0 \pm 4.2 \mathrm{mg}$ ), respectively.

[Conclusion] MMF is effective treatment for renal and nonrenal disease manifestation in Japanese patients with SLE. We could not find significant differences for efficacy among the treatments. 